\title{
Theory of phase-sensitive measurement of photon-assisted tunneling through a
} quantum dot

\section{Jauho, Antti-Pekka; Wingreen, Ned S.}

\section{Published in:}

Physical Review B

Link to article, DOI:

10.1103/PhysRevB.58.9619

Publication date:

1998

Document Version

Publisher's PDF, also known as Version of record

Link back to DTU Orbit

Citation (APA):

Jauho, A-P., \& Wingreen, N. S. (1998). Theory of phase-sensitive measurement of photon-assisted tunneling through a quantum dot. Physical Review B, 58(15), 9619-9622. https://doi.org/10.1103/PhysRevB.58.9619

\section{General rights}

Copyright and moral rights for the publications made accessible in the public portal are retained by the authors and/or other copyright owners and it is a condition of accessing publications that users recognise and abide by the legal requirements associated with these rights.

- Users may download and print one copy of any publication from the public portal for the purpose of private study or research.

- You may not further distribute the material or use it for any profit-making activity or commercial gain

- You may freely distribute the URL identifying the publication in the public portal

If you believe that this document breaches copyright please contact us providing details, and we will remove access to the work immediately and investigate your claim. 


\title{
Theory of phase-sensitive measurement of photon-assisted tunneling through a quantum dot
}

\author{
A. P. Jauho \\ Mikroelektronik Centret, Technical University of Denmark, Bldg 345 East, DK-2800 Lyngby, Denmark
}

Ned S. Wingreen

NEC Research Institute, 4 Independence Way, Princeton, New Jersey 08540

(Received 15 January 1998)

\begin{abstract}
Recent double-slit interference experiments [Schuster et al., Nature (London) 385, 417 (1997)] have demonstrated the possibility of probing the phase of the complex transmission coefficient of a quantum dot via the Aharonov-Bohm effect. We propose an extension of these experiments: an ac voltage imposed on the side gate with the concomitant photonic sidebands leads to additional structure both in the amplitude and in the phase of the Aharonov-Bohm signal. Observation of these effects would be a definitive proof of coherent absorption and reemission of photons from the ac source. [S0163-1829(98)08736-0]
\end{abstract}

Phase coherence is the hallmark of all mesoscopic transport phenomena. Yet normal transport measurements yield information only about the magnitude of the transmission amplitude, and not its phase. In a groundbreaking set of experiments, Yacoby et al. ${ }^{1}$ and Schuster et al. ${ }^{2}$ recently demonstrated that a phase measurement is nevertheless possible in a mesoscopic double-slit geometry. Their experimental protocol can be summarized as follows: A magnetotransport measurement is performed on an Aharonov-Bohm ring with a quantum dot fabricated in one of its arms. If the quantum dot supports coherent transport, the transmission amplitudes through the two arms interfere. A magnetic field induces a relative phase change $2 \pi \Phi / \Phi_{0}$ between the two transmission amplitudes $t_{0}$ and $\widetilde{t}_{Q D}$, leading to an oscillatory component to the conductance $g(B)=\left(e^{2} / h\right) \mathcal{T}(B)$, with

$$
\mathcal{T}(B)=\mathcal{T}^{(0)}+2 \operatorname{Re}\left\{t_{0}^{*} \tilde{t}_{Q D} e^{2 \pi i \Phi / \Phi_{0}}\right\}+\cdots,
$$

where $\Phi$ is the flux threading the ring, $\Phi_{0}=h c / e$ is the flux quantum, and where the ellipsis represents higher harmonics due to multiple reflections. The amplitudes $t_{0}$ and $\widetilde{t}_{Q D}$ give the coherent parts of the two sets of paths joining the emitter and the collector; the incoherent components lead to a structureless background signal, which can be neglected in the forthcoming analysis. In the experiments, an oscillatory component in magnetoconductance of this form was clearly observed thus demonstrating coherent transmission through the arm with the dot. ${ }^{1,2}$ In the experiment of Yacoby et al., ${ }^{1}$ the Aharonov-Bohm phase could take on only two values 0 and $\pi$ as a consequence of microreversibility in a two-terminal geometry. ${ }^{3,4}$ The second generation of experiments, ${ }^{2}$ in a four-terminal geometry, allowed a determination of the continuous phase shift of the transmission amplitude through the dot. The success of these experiments gave rise to a number of other works which concentrated on refining the interpretation of the experimental results. ${ }^{3-6}$ Yet, the experiments also suggest application to other phase-coherent transport processes. One particular example, which has been of considerable recent interest both experimentally ${ }^{7-11}$ and theoretically, ${ }^{12-17}$ is photon-assisted tunneling. While photon-assisted tunneling (PAT) is intrinsically a coherent phenomenon, existing measurements of PAT are insensitive to the phase of the transmitted electrons, and do not directly demonstrate coherence in the presence of the time-dependent field. Here we propose a measurement of photon-assisted tunneling through a quantum dot in the mesoscopic doubleslit geometry described above (see Fig. 1). In essence, we propose a combination of the experiments of Kouwenhoven and co-workers, ${ }^{8,11}$ where a microwave-modulated side-gate

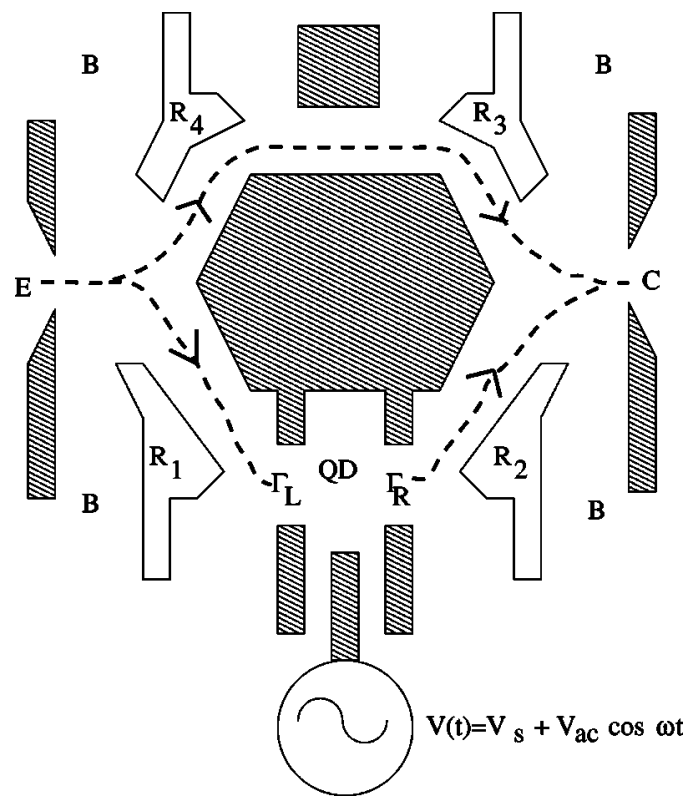

FIG. 1. Schematic layout of the proposed multiterminal doubleslit interference experiment. The device consists of an AharonovBohm ring with a quantum dot (QD) in one arm, defined with metallic gates (shaded areas) on the 2D electron gas. Electron paths (shown as dashed lines) originating from the emitter $(E)$ interfere at the collector $(C)$. A time-dependent voltage $V(t)$ is applied to the quantum dot via a side gate. The reflector gates $R_{1 \cdots 4}$, shown as white areas, direct multiply reflected paths to the common base $(B)$, thus preventing them from contributing to the interference signal, in accordance with Eq. (1). 
voltage gave rise to photon-assisted tunneling through a quantum dot, and the interference experiments of Refs. 1 and 2.

For an experiment of this type, we calculate the coherent transmission amplitude through the quantum dot in the presence of an arbitrarily strong ac potential applied to the side gate. Our theoretical results indicate that phase-coherent absorption and reemission of photons can be unambiguously demonstrated via phase measurements at the sidebands of the main transmission resonance. In addition, for large driving amplitudes the phase shift associated with the main transmission resonance can be reversed from its usual behavior, providing a direct demonstration of coherence in a strong ac potential.

We focus on transport in the neighborhood of a single Coulomb oscillation peak associated with a single nondegenerate electronic level of the quantum dot. ${ }^{18}$ The effect of the ac side-gate voltage is described entirely through the timedependent energy of this level,

$$
\epsilon(t)=\epsilon_{0}\left(V_{s}\right)+V_{\mathrm{ac}} \cos \omega t,
$$

where the static energy of the level $\epsilon_{0}$ depends on the dc side-gate voltage $V_{s}$. All other levels on the dot can be neglected provided the ac amplitude $V_{\mathrm{ac}}$ and the photon energy, $\hbar \omega$ are small compared to the level spacing on the dot.

The energy dependence of the coherent part of the transmission amplitude $\tilde{t}_{Q D}(\epsilon)$ through the arm containing the quantum dot is determined by the transmission amplitude $t_{Q D}(\epsilon)$ through the dot, $\tilde{t}_{Q D}(\epsilon) \propto t_{Q D}(\epsilon)$. In the absence of an ac potential, a suitable model for the dot transmission amplitude is the Breit-Wigner form

$$
t_{Q D}(\epsilon)=\frac{-i \sqrt{\Gamma_{L} \Gamma_{R}}}{\epsilon-\epsilon_{0}\left(V_{s}\right)+i \Gamma / 2},
$$

where $\Gamma=\Gamma_{L}+\Gamma_{R}$ is the full width at half maximum of the resonance on the dot due to tunneling to the left and right leads. Equation (3) implies a continuous phase accumulation of $\pi$ in the transmission amplitude as the Coulomb blockade peak is traversed. (Note that the Breit-Wigner form is exact for a noninteracting system with $\Gamma$ independent of energy.)

In the dynamic case, the simple Breit-Wigner description must be generalized, and the object to evaluate is the $S$-matrix element. ${ }^{19,20}$ Provided interactions in the leads can be neglected, the elastic transmission amplitude $t_{Q D}(\epsilon)$ can be written as the energy-conserving part of the $S$ matrix between the left and right leads,

$$
\lim _{\epsilon^{\prime} \rightarrow \epsilon}\left\langle\epsilon^{\prime}, R|\mathcal{S}| \epsilon, L\right\rangle=\delta\left(\epsilon^{\prime}-\epsilon\right) t_{Q D}(\epsilon) .
$$

The $S$ matrix is simply related to the retarded Green function of the level on the dot, including both tunneling to the leads and the ac potential, ${ }^{19}$

$$
\left\langle\epsilon^{\prime}, R|\mathcal{S}| \epsilon, L\right\rangle=-i \frac{\sqrt{\Gamma_{L} \Gamma_{R}}}{2 \pi} \iint d t d t_{1} e^{i\left(\epsilon^{\prime} t-\epsilon t_{1}\right)} G^{r}\left(t, t_{1}\right) .
$$

Combining Eqs. (4) and (5) allows us to write

$$
t_{Q D}(\epsilon)=-i \sqrt{\Gamma_{L} \Gamma_{R}}\langle A(\epsilon, t)\rangle_{t},
$$

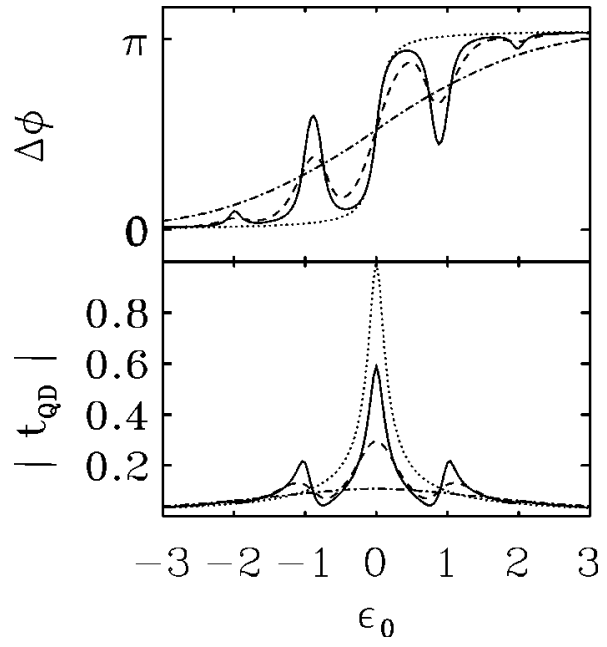

FIG. 2. Temperature dependence of the phase shift $\Delta \phi$ (top panel) and the square of the amplitude (bottom) of $t_{Q D}$. The level width is $\Gamma / 2=0.1$, in terms of which the other parameters are $V_{\mathrm{ac}}$ $=1.0, \omega=1.0$, and $T=0$ (solid line), 0.1 (dashed line), and 0.5 (dash-dotted line). For comparison, the $T=0$ time-independent results are shown as dots, cf. Eq. (3).

where the brackets denote a time average, and where

$$
A(\epsilon, t)=\int d t_{1} e^{i \epsilon\left(t-t_{1}\right)} G^{r}\left(t, t_{1}\right) .
$$

For the time-dependent energy level given by Eq. (2), we find $^{20}$

$$
G^{r}\left(t, t_{1}\right)=-i \theta\left(t-t_{1}\right) \exp \left[-\frac{\Gamma}{2}\left(t-t_{1}\right)-i \int_{t_{1}}^{t} d t^{\prime} \epsilon\left(t^{\prime}\right)\right],
$$

so that

$$
\langle A(\epsilon, t)\rangle_{t}=\sum_{k=-\infty}^{\infty} \frac{J_{k}^{2}\left(V_{\mathrm{ac}} / \hbar \omega\right)}{\epsilon-\epsilon_{0}\left(V_{s}\right)-k \hbar \omega+i \Gamma / 2} .
$$

A combination of Eqs. (6) and (9), evaluated at the Fermi energy, gives the relevant transmission amplitude, and hence the amplitude of the Aharonov-Bohm oscillations at $T$ $=0 \mathrm{~K}$. At finite temperatures one must compute $t_{Q D}=\int d \epsilon$ $\left(-\partial f_{0} / \partial \epsilon\right) t_{Q D}(\epsilon)$, where $f_{0}(\epsilon)$ is the Fermi function, and the final result is

$$
\begin{aligned}
t_{Q D}= & \left(-\frac{\Gamma}{4 \pi T}\right) \sum_{k=-\infty}^{\infty} J_{k}^{2}\left(V_{\mathrm{ac}} / \hbar \omega\right) \\
& \times \psi^{\prime}\left[\frac{1}{2}-\frac{i}{2 \pi T}\left(\mu-\epsilon_{0}\left(V_{s}\right)-k \hbar \omega+i \frac{\Gamma}{2}\right)\right],
\end{aligned}
$$

where $\psi^{\prime}$ is the derivative of the digamma function, and $\mu$ is the chemical potential in the leads.

Equation (10) is the main result of this paper, and in what follows we shall evaluate it in several cases of interest. We emphasize that a conventional conductance measurement would yield information only about the time average of the square of the transmission amplitude, and the double-slit geometry is necessary in order to probe the phase. Figure 2 shows the computed magnitude of $t_{Q D}$ (bottom) and its 


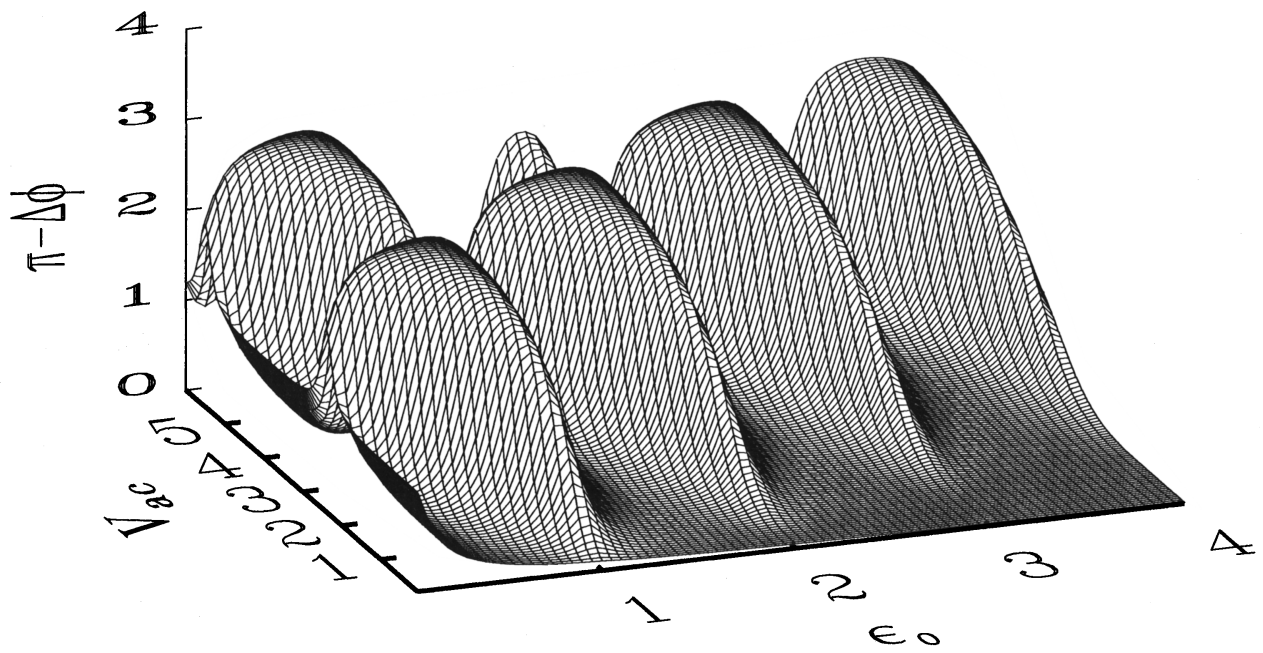

(a)

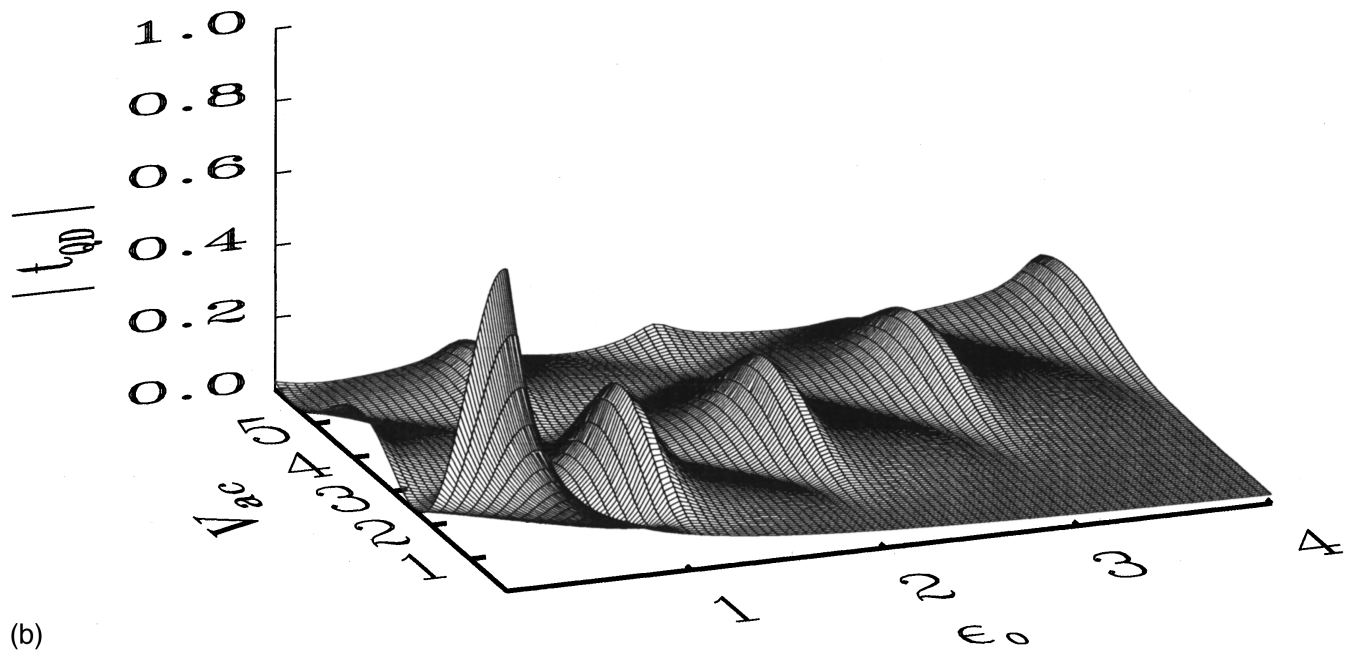

FIG. 3. The phase shift $\pi-\Delta \phi$ (top panel) and the square of the amplitude (bottom) for $\omega=1.0, \Gamma / 2=0.1$, and $T=0$. The energy axis corresponds to $\epsilon_{0}\left(V_{s}\right)$ with $\mu=0$.

phase (top), as a function of the level energy $\epsilon_{0}\left(V_{s}\right)$. As compared to the time-independent case (shown as a dotted line), several features are noteworthy. The magnitude of $t_{Q D}$ shows photonic sidebands, reminiscent of those seen in transmission through a microwave modulated quantum dot. ${ }^{8}$ However, there is an important difference from the usual case of photon-assisted tunneling. The amplitude of the Aharonov-Bohm oscillation is sensitive only to the time average of the transmission amplitude $t_{Q D}$. Hence only elastic transmission through the dot contributes, i.e., the net number of photons absorbed from the ac field must be zero. The sideband at, say, $\epsilon=\epsilon_{0}\left(V_{s}\right)-\hbar \omega$ corresponds to a process in which an electron first absorbs a photon to become resonant at energy $\epsilon_{0}\left(V_{s}\right)$, and subsequently reemits the photon to return to its original energy.

Perhaps most interesting are the features appearing in the phase: the phase shift shows a nonmonotonic behavior, with pronounced resonances located at the energies corresponding to the photonic sidebands. The strengths of these phase resonances are strongly dependent on the ac amplitude $V_{\mathrm{ac}}$, and in Fig. 3 we show the computed signal as a function of both $\epsilon_{0}\left(V_{s}\right)$ and the amplitude of modulation. In Fig. 4 we highlight another interesting consequence of Eq. (10): it is pos-

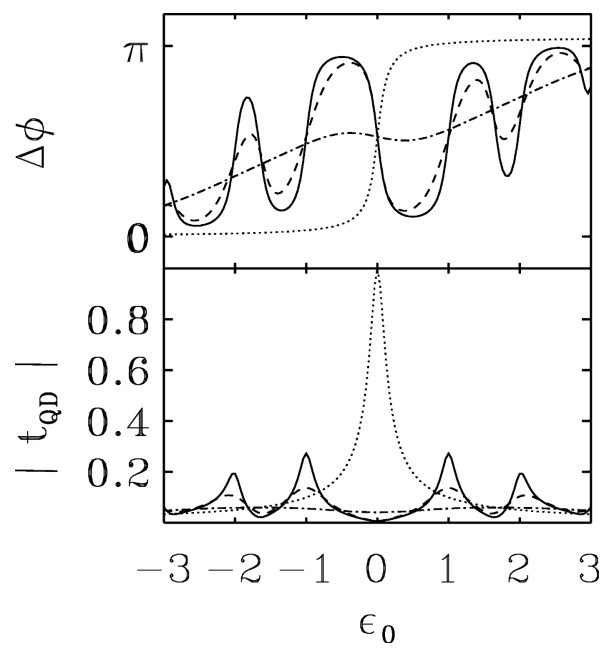

FIG. 4. Temperature dependence of the phase shift (top panel) and the amplitude (bottom) of $t_{Q D}$, for $V_{\mathrm{ac}}=2.405, \omega=1.0, \Gamma / 2$ $=0.1$, and $T=0,0.1$, and 0.5, with line types as in Fig. 2. Note the qualitative differences as compared to Fig. 2: suppression of the main transmission peak, and the negative slope of the phase shift at $\epsilon_{0}=0$. 
sible to quench the main transmission peak (bottom panel) entirely, ${ }^{21}$ or change the sign of the slope of the phase at resonance by adjusting the ratio $V_{\text {ac }} / \hbar \omega$ to coincide with a zero of the Bessel function $J_{0}$ (top). This phenomenon is mathematically analogous to the recently observed absolute negative conductivity in THz-irradiated superlattices; ${ }^{9}$ in our case, however, it is the phase rather than the current that displays this behavior.

In summary, we have proposed an experiment to probe phase coherence in a quantum dot driven by a strong ac potential. The phase measurement relies on the mesoscopic double-slit geometry pioneered in Refs. 1 and 2 . The amplitude of Aharonov-Bohm oscillations reflects the amplitude for coherent transmission through the dot with zero net absorption of photons. We find that coherent absorption and reemission of photons can be unambiguously detected via phase measurement at sidebands of the main transmission resonance through the quantum dot.

The authors acknowledge useful comments from Karsten Flensberg, Ben Yu-Kuang Hu, and Andreas Wacker.
${ }^{1}$ A. Yacoby, M. Heiblum, D. Mahalu, and H. Shtrikman, Phys. Rev. Lett. 74, 4047 (1995).

${ }^{2}$ R. Schuster, E. Buks, M. Heiblum, D. Mahalu, V. Umansky, and H. Shtrikman, Nature (London) 385, 417 (1997).

${ }^{3}$ A. Levy Yeyati and M. Büttiker, Phys. Rev. B 52, R14 360 (1995).

${ }^{4}$ A. Yacoby, R. Schuster, and M. Heiblum, Phys. Rev. B 53, 9583 (1995).

${ }^{5}$ G. Hackenbroich and H. A. Weidenmüller, Phys. Rev. Lett. 76, 110 (1996); Phys. Rev. B 53, 16379 (1996).

${ }^{6}$ C. Bruder, R. Fazio, and H. Schoeller, Phys. Rev. Lett. 76, 114 (1996).

${ }^{7}$ P. S. S. Guimarães, B. J. Keay, J. P. Kaminsky, S. J. Allen, Jr., P. F. Hopkins, A. C. Gossard, L. T. Florez, and J. P. Harbison, Phys. Rev. Lett. 70, 3792 (1993).

${ }^{8}$ L. Kouwenhoven, S. Jauhar, J. Orenstein, P. L. McEuen, Y. Nagamune, J. Motohisa, and H. Sakaki, Phys. Rev. Lett. 73, 3443 (1993).

${ }^{9}$ B. J. Keay, S. Zeuner, S. J. Allen, Jr., K. D. Maranowski, A. C. Gossard, U. Bhattacharya, and M. J. W. Rodwell, Phys. Rev. Lett. 75, 4102 (1995).

${ }^{10}$ S. Zeuner, B. J. Keay, S. J. Allen, Jr., K. D. Maranowski, A. C. Gossard, U. Bhattacharya, and M. J. W. Rodwell, Phys. Rev. B 53, 1717 (1996). A detailed theoretical analysis of these experi- ments is presented in A. Wagner, A.-P. Jauho, S. Zeuner, and S. J. Allen, ibid. 56, 13268 (1997); note, however, that the tunneling in these experiments is sequential, and would not support the interference effects discussed in the present paper.

${ }^{11}$ T. H. Oosterkamp, L. P. Kouwenhoven, A. E. A. Koolen, N. C. van der Vaart, and C. J. P. M. Harmans, Phys. Rev. Lett. 78, 1536 (1997).

${ }^{12}$ D. Sokolovski, Phys. Rev. B 37, 4201 (1988).

${ }^{13}$ C. Bruder and H. Schöller, Phys. Rev. Lett. 72, 1076 (1994).

${ }^{14}$ M. Wagner, Phys. Rev. Lett. 76, 4010 (1996).

${ }^{15}$ J. Inarrea and G. Platero, Europhys. Lett. 34, 43 (1996).

${ }^{16}$ T. H. Stoof and Yu. V. Nazarov, Phys. Rev. B 53, 1050 (1996).

${ }^{17}$ C. A. Stafford and N. S. Wingreen, Phys. Rev. Lett. 76, 1916 (1996).

${ }^{18}$ U. Meirav and E. B. Foxman, Semicond. Sci. Technol. 10, 255 (1995).

${ }^{19}$ N. S. Wingreen, K. W. Jacobsen, and J. W. Wilkins, Phys. Rev. B 40, 11834 (1989).

${ }^{20}$ N. S. Wingreen, A. P. Jauho, and Y. Meir, Phys. Rev. B 48, 8487 (1993); A. P. Jauho, N. S. Wingreen, and Y. Meir, Phys. Rev. B 50, 5528 (1994).

${ }^{21} \mathrm{~A}$ detailed analysis of the zero-temperature quenching of transmission through an ac-modulated quantum well is available in M. Wagner, Phys. Rev. B 49, 16544 (1994). 